\title{
Review of: "Adaptive learning and recall of motor- sensory sequences in adult echolocating bats"
}

\author{
Chung-Hsin $\mathrm{Wu}^{1}$ \\ 1 National Taiwan Normal University
}

Potential competing interests: The author(s) declared that no potential competing interests exist.

Review of for"Adaptive learning and recall of motor-sensory sequences in adult echolocating bats"

The paper shows that Pipistrellus kuhlii bats can gradually adapt their pre-takeoff echolocation sequence when moved to a constantly cluttered environment. The results demonstrated long-term adaptive flexibility in sensory acquisition in adult animals. In addition, the paper gives further insight into the importance of sensory planning in the initiation of a precise sensorimotor behavior such as approaching for landing. Little is known about animals' abilities to adapt their sensing during life. In this study, they demonstrate long-term plasticity in sensory acquisition in adult bats. Their results indicated that adaptive learning over long-time periods can enhance sensory planning before initiation of an approach when adapting to a new environment. 